CERN-TH/2001-025

KEK-TH-743

MSUHEP-01130

\title{
Single production of charged Higgs bosons at linear colliders ${ }^{1}$
}

\author{
S. Kanemura ${ }^{a}$, S. Moretti ${ }^{b}$ and K. Odagiri ${ }^{c}$ \\ ${ }^{a}$ Physics and Astronomy Department, Michigan State University, East Lansing, MI 48824-1116, \\ USA \\ ${ }^{b}$ CERN, CH 1211 Geneva 23 Switzerland \\ ${ }^{c}$ Theory Group, KEK, 1-1 Oho, Tsukuba, Ibaraki 305-0801, Japan
}

\begin{abstract}
We discuss single charged Higgs boson production from $e^{+} e^{-}$annihilation at next generation linear colliders. They can be important to study the phenomenology of charged Higgs bosons, especially when charged Higgs bosons are too heavy to be produced by the pair production mechanism. Cross sections for various single production processes are evaluated at the leading order. Our analysis shows that in some parameter regions the phenomenology of charged Higgs bosons can be explored even beyond the kinematic limit for pair production by using single production processes.
\end{abstract}

\section{INTRODUCTION}

Electroweak symmetry breaking sectors which are composed of two scalar isospindoublets predict charged Higgs bosons $\left(H^{ \pm}\right)$. Their discovery at future colliders will directly indicate such a non-minimal structure of the Higgs sector, and its detailed information will give strong hints for the structre of physics beyond the Standard Model (SM). Therefore, their phenomenology has been evaluated especially over the past few years as their discovery potential has become more clear.

At CERN Large Hadron Collider, charged Higgs bosons below the top quark mass will be produced in the top quark decay process. For heavier charged Higgs bosons, they will be produced mainly through the subprocesses $g b \rightarrow t H^{-}, g g \rightarrow t \bar{b} H^{-}$and their charge conjugate, and they may be probed, for example, using the decay modes $H^{-} \rightarrow \tau^{-} \bar{\nu}$ and its charge conjugate especially for large $\tan \beta$ values, in spite of huge QCD backgrounds.

1) Talk given by S. Kanemura at the Linear Collider Workshop 2000, October 24-28, 2000, Fermilab, USA. 
On the other hand, at future electron-positron linear colliders (LC's), the dominant production process should be the pair production [1] $e^{-} e^{+} \rightarrow H^{+} H^{-}$. Its cross section depends only on the charged Higgs mass, and this process provides a hallmark channel through which we can study $H^{ \pm}$phenomenology [2] as long as the charged Higgs boson mass $\left(M_{H^{ \pm}}\right)$is sufficiently smaller than its kinematical threshold $\sqrt{s} / 2$. When $M_{H^{ \pm}}$is near or above $\sqrt{s} / 2$, the pair production is not available. In such a case, the phenomenology of charged Higgs bosons may be explored through the kinematically-allowed single production processes.

In this talk, we discuss various single $H^{ \pm}$production channels to complement the pair production channel. We consider the following processes [3]:

$$
\begin{aligned}
& e^{-} e^{+} \rightarrow \tau^{-} \bar{\nu}_{\tau} H^{+}, \tau^{+} \nu_{\tau} H^{-} \\
& e^{-} e^{+} \rightarrow \bar{t} b H^{+}, t \bar{b} H^{-} \\
& e^{-} e^{+} \rightarrow W^{\mp} H^{ \pm} \text {(one loop) } \\
& e^{-} e^{+} \rightarrow e^{-} \bar{\nu} H^{+}, e^{+} \nu H^{-} \text {(one loop) } \\
& e^{-} e^{+} \rightarrow Z^{0} W^{\mp} H^{ \pm} \\
& e^{-} e^{+} \rightarrow h^{0} W^{\mp} H^{ \pm} \\
& e^{-} e^{+} \rightarrow H^{0} W^{\mp} H^{ \pm} \\
& e^{-} e^{+} \rightarrow A^{0} W^{\mp} H^{ \pm} \\
& e^{-} e^{+} \rightarrow e^{-} e^{+} W^{\mp} H^{ \pm} \\
& e^{-} e^{+} \rightarrow \nu_{e} \bar{\nu}_{e} W^{\mp} H^{ \pm} \\
& e^{-} e^{+} \rightarrow e^{-} \bar{\nu}_{e} Z^{0} H^{+}, e^{+} \nu_{e} Z^{0} H^{-} \\
& e^{-} e^{+} \rightarrow e^{-} \bar{\nu}_{e} h^{0} H^{+}, e^{+} \nu_{e} h^{0} H^{-} \\
& e^{-} e^{+} \rightarrow e^{-} \bar{\nu}_{e} H^{0} H^{+}, e^{+} \nu_{e} H^{0} H^{-} \\
& e^{-} e^{+} \rightarrow e^{-} \bar{\nu}_{e} A^{0} H^{+}, e^{+} \nu_{e} A^{0} H^{-} .
\end{aligned}
$$

The $\tau \nu_{\tau}$ associated production mode (1) has been studied in the literature [4]. The processes (2), (6), (7) and (8), as well as (5) with $Z \rightarrow b \bar{b}$ lead to the same final state $b \bar{b} W^{ \pm} H^{\mp}[5]$. The one-loop induced processes $e^{-} e^{+} \rightarrow H^{ \pm} W^{\mp}$ have also been studied in Refs. [6,7]. The one-loop induced $H W Z$ and $H W \gamma$ vertices which enter into (4) have been calculated in Ref. [8].

\section{RESULTS}

We evaluate cross sections of the above single production processes at the leading order. The Two-Higgs-Doublet-Model (2HDM) parameters are taken assuming the Minimal Supersymmetric Standard Model. For the SM parameters we adopted the following numbers: $m_{b}=4.25 \mathrm{GeV}, m_{t}=175 \mathrm{GeV}, m_{e}=0.511 \mathrm{MeV}, m_{\tau}=1.78$ $\mathrm{GeV}, m_{\nu}=0, M_{W}=80.23 \mathrm{GeV}, \Gamma_{W}=2.08 \mathrm{GeV}, M_{Z}=91.19 \mathrm{GeV}, \Gamma_{Z}=2.50$ $\mathrm{GeV}, \sin ^{2} \theta_{W}=0.232$. The Center-of-Mass energy of the collider $(\sqrt{s})$ is assumed 

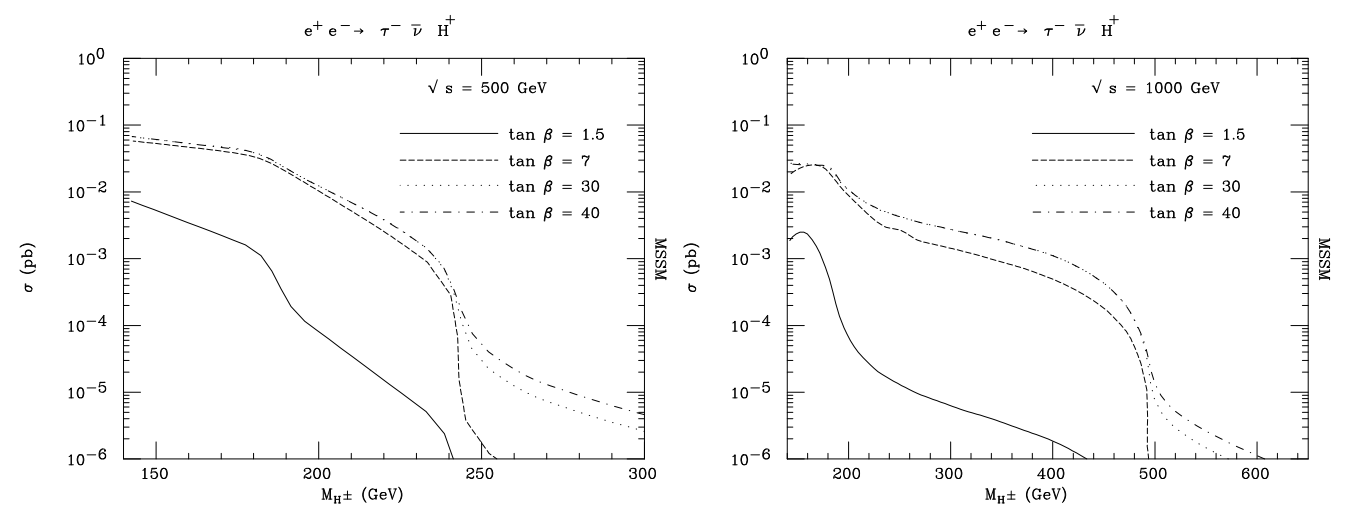

FIGURE 1. Total cross sections for the tau associated production channels (1).
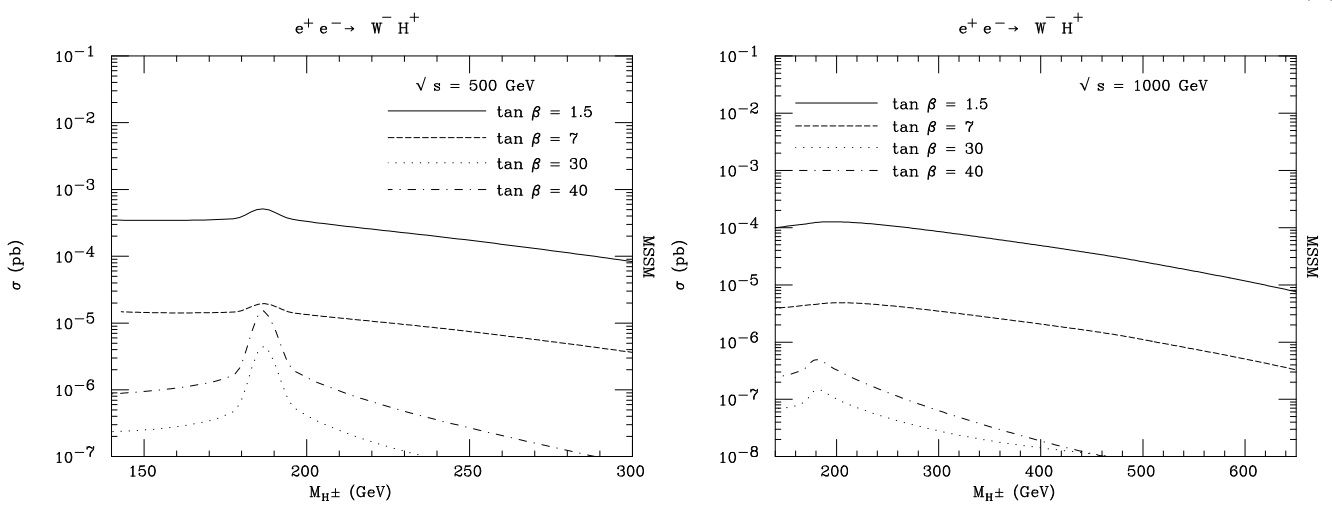

FIGURE 2. Total cross sections for the $W^{-} H^{+}$associated production process (3).

to be $500 \mathrm{GeV}$ and $1000 \mathrm{GeV}$. Details of the calculation and the plots are shown in Ref. [3].

The $\tau \nu_{\tau}$ associated production processes (1), shown in Fig. 1, are dominated by the pair production as long as it is kinematically allowed $\left(M_{H^{ \pm}}<\sqrt{s} / 2\right)$. Above this threshold, the cross sections still exceed $10^{-5} \mathrm{pb}$ at large $\tan \beta$ values. If $H^{ \pm}$is heavy enough to decay into $t b$, dominant background contribution comes from topquark pair production. By peforming $W$ and $t$ mass reconstruction, the background can be reduced naively by $\mathcal{O}\left(\alpha_{\mathrm{EW}}^{2}\right)$, so that the signal would be visible, although detailed simulations are needed depending on each machine.

The $t \bar{b}$ (or $\bar{t} b$ ) associated production process (2) has a similar structure to $\tau \nu_{\tau} H^{ \pm}$ modes, but the $\tan \beta$ dependence is opposite: the rate is larger for smaller $\tan \beta$ values. The cross section is suppressed above the pair production threshold in comparison with that of $\tau \nu_{\tau} H^{ \pm}$modes because of the difference in the phase space.

Figure 2 shows the cross section of the one-loop induced processes $e^{-} e^{+} \rightarrow$ $W^{ \pm} H^{\mp}(3)$. For small $\tan \beta$ values, the $H^{ \pm} t b$ coupling included in each quarkloop diagram is large so that the cross section becomes substantial: the $\tan \beta$ dependence of the cross section is $\sim m_{t}^{4} \cot ^{2} \beta$ at small $\tan \beta$ and $\sim m_{b}^{4} \tan ^{2} \beta$ at 
very large $\tan \beta$. The cross section remains large beyond the pair production kinematic limit. When the dominant $H^{ \pm}$decay mode is $t \bar{b}$, we can deal with the top pair background by reconstructing the final state and eliminating events with two top quarks. This is expected to reduce the top pair background by about $\alpha_{\mathrm{EW}}$, which should enable the observation of the peak at the charged Higgs mass in some regions of the parameter space.

For the rest of the processes, (4)-(14), there is a variety of structures observed in the cross sections. Their rates, however, turned out to be very small for larger $M_{H^{ \pm}}$ than the pair production threshold. This is owing to the reasons such as coupling suppression, cancellation between Feynman diagrams, and/or smaller phase space. Details of the numerical results are shown in Ref. [3]. As mentioned, the processes (2), (5), (6), (7) and (8) lead to the final state $b \vec{b} H^{ \pm} W^{\mp}$. Some channels have large cross sections for $M_{H^{ \pm}}<\sqrt{s} / 2$ [3]. The variety of resonance structures imply little interference among these processes. The $e^{-} e^{+} \rightarrow b \bar{b} H^{ \pm} W^{\mp}$ modes, therefore, complement the pair production in the charged Higgs study at LC especially below the threshold of the pair production [5]. The procedure for the tagging of the final state $b \bar{b} H^{ \pm} W^{\mp}$ is outlined in Ref. [3].

In either process, information of the tau [9] and the top [10] polarization can be used to help identify the charged Higgs boson.

\section{CONCLUSION}

We found that the $\tau \bar{\nu}_{\tau} H^{+}$channel and the loop induced $H^{ \pm} W^{\mp}$ channel are the most promising channels for studying charged Higgs phenomenology beyond the kinematic limit for pair production. The $H^{ \pm} W^{\mp}$ channel is enhanced at low $\tan \beta$, whereas the $\tau \bar{\nu}_{\tau} H^{+}$channel is enhanced at large $\tan \beta$. For the charged Higgs bosons whose mass is below the pair production threshold, some of the single production channels which lead to the $b \bar{b} H^{ \pm} W^{\mp}$ final state complement the pair production. Some of their cross sections become large, but they are rapidly suppressed above the kinematic limit of the pair production. Our results motivate further study of the decay modes, the hadronic final states and the backgrounds relevant to these charged Higgs boson single production processes.

\section{REFERENCES}

1. S. Komamiya, Phys. Rev. D38 (1988) 2158.

2. A. Kiiskinen, M. Battaglia and P. Pöyhönen, these proceedings.

3. S. Kanemura, S. Moretti and K. Odagiri, hep-ph/0012030.

4. A. Gutierrez-Rodriguez and O.A. Sampayo, hep-ph/9911361.

5. S. Moretti and K. Odagiri, Eur. Phys. J. C1 (1998) 633.

6. A. Arhrib, M. Capdequi Peyranère, W. Hollik and G. Moultaka, Nucl. Phys. B581 (2000) 34; S.H. Zhu, hep-ph/9901221.

7. S. Kanemura, Eur. Phys. J. C17 (2000) 473. 
8. T.G. Rizzo, Mod. Phys. Lett. A4 (1989) 2757; A. Mendez and A. Pomarol, Nucl. Phys. B349 (1991) 369; M. Capdequi Peyranère, H.E. Haber and P. Irulegui, Phys. Rev. D44 (1991) 191; S. Kanemura, Phys. Rev. D61 (2000) 095001.

9. B.K. Bullock, K. Hagiwara and A.D. Martin, Phys. Lett. B67 (1991) 3055.

10. K. Odagiri, Phys. Lett. B452 (1999) 327. 